\title{
Ketamine in the treatment of Major Depressive Disorder and its
}

Potential for Abuse

D. Vilaverde Freitas $^{1}$, R. Teixeira Ribeiro ${ }^{1}$, C. Pedro ${ }^{1}$, C. Soares ${ }^{1}$, B. Jorge ${ }^{1}$, S. Carneiro ${ }^{1}$. ${ }^{1}$ Hospital de Braga, Psychiatry Department, Braga, Portugal.

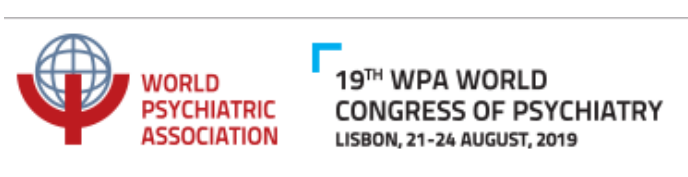

Objectives:

A literature review about the potential of abuse with ketamine in the treatment of Major Depressive Disorder (MDD).

\section{Background:}

Ketamine is a non-selective $\mathrm{N}$-methyl-D-aspartate (NMDA) receptor antagonist, that also has effect on $\mathrm{k}$-opioid and sigma receptors. ${ }^{(1)}$ It has been used since 1970 as a dissociative anesthetic and gained popularity as a recreational drug in 1990.(2) People misusing ketamine may report experiences of dissociation, derealization, depersonalization and hallucinations. (2)

There is no data on the prevalence of ketamine nonmedical use, but its misuse has been increasing in the past decade and its illicit use poses major public health challenges in many countries. ${ }^{(1,3)}$

Ketamine reinforcing effects are thought to be dopamine mediated, with rapid development of tolerance, thus leading to its potential for abuse and dependence. ${ }^{(4)}$ It is a regulated substance in many countries for its liability for abuse. ${ }^{(5)}$.

Besides this potential for abuse, urinary tract lesions and cognitive impairment are described in long-term use of ketamine. (6,7)

Since 2000, ketamine is being studied as a potential treatment in Major Depressive Disorder (MDD), due to its modulation of glutaminergic system which is thought to be involved in depression pathophysiology. ${ }^{(8)}$

\section{Materials and Methods:}

The literature search was developed in the MEDLINE/Pubmed for relevant articles, using keywords for all variants of "ketamine", "depression" and "abuse".

\section{Results:}

Several studies show that the use of a single infusion of subanesthetic dosing of ketamine reduces depressive symptoms and suicidal ideation in patients with refractory MDD within 4 hour to 1 day after administration. ${ }^{(9-13)}$ These benefits seem to last 3-14 days after administration, ${ }^{(9,13-}$ 16) with relapse of symptoms being reported in some studies after treatment cessation, even in patients with repeated administrations of ketamine.(17-20)

With this growing evidence of a rapid antidepressant effect, numerous private clinics started offering ketamine as an off-label treatment for refractory depression, although the safety of repeated infusions of ketamine has not been fully investigated in large clinical studies(21)

A systematic review that evaluated the side effects of ketamine use in depression, reported that short term psychiatric, psychotomimetic, cardiovascular and neurological effects were more frequently reported with ketamine than placebo but these were transient and associated with acute intravenous administration of ketamine. ${ }^{(22)}$ Conclusions could not be drawn for repeated doses of ketamine, since insufficient data was available regarding the side effects of repeated dosing and possible cumulative and long-term risks, as known in other patient groups exposed to ketamine.(22)

Two case reports described a development of ketamine dependence in persons who presented MDD. One paper described the case of a 52year-old man with a history of recurrent MDD, persistent depressive disorder and a previous treatment for alcohol use disorder.(23) The patient had been enrolled in a study with a single infusion of ketamine, with improvement in depressive symptoms. The patient then searched for a ketamine prescriber and started using ketamine beyond the prescribed recommendations, developing a ketamine dependence.(23) Another case report described the history of a 50 -year-old anesthetic nurse that developed ketamine dependence after self-administrating increasing doses of intramuscular ketamine in order to treat her depressive disorder. ${ }^{(24)}$ This case also points to a probable development of tolerance in ketamine's antidepressant response.(24)

\section{Discussion:}

In order to approve a new treatment, there must be evidence not only of its efficacy but also of its safety, with a favorable benefit to risk ratio. There are some examples of drugs developed to treat medical conditions that, after approval for prescription, resulted in public health crises due to its addictive features, as for instance oxycodone, that outweighed their clinical benefits. ${ }^{(25,26)}$

Since the risk of substance abuse with repeated administrations of ketamine in the treatment of MDD patients remains unclear, it is important that clinicians, regulatory agencies and pharmaceutical companies, work together to establish a suitable framework for its therapeutic use before ketamine reaches widespread clinical use. (26) For instance, the use of ketamine enantiomer - esketamine - was approved by the FDA for treatment-resistant MDD this year, but due to concerns with liability for abuse is only available through a restricted program under a Risk Evaluation and Mitigation Strategy. (27)

Also, two other aspects should be considered in treating MDD patients with ketamine when considering its known potential for abuse. Firstly, there is frequently comorbidity of MDD with substance abuse disorders, which could hypothetically potentiate the risk of abuse with ketamine. (23) Studies often exclude subjects with history of substance use disorder and the effects of ketamine on this population are not known. Secondly, the use of ketamine in MDD patients, seems to provoke rapid but transient symptom relieve, which could contribute to seeking behavior of ketamine in order to alleviate symptoms in non-clinical settings, as described in the two case reports.(5)

\section{Conclusion:}

Although ketamine treatment has been shown to be effective by rapidly reducing or remitting depressive symptoms and suicidal ideation in treatment resistant MDD, the adequate length and frequency of treatment it is not yet defined.

Also, long-term outcomes or consequences of repeated use of ketamine were not systematically studied in these population, namely its potential for abuse.

Further studies that address these problems are needed, in order to establish specific clinical indications and regulatory measures for this treatment before widespread off-label use of ketamine in the treatment of MDD in non-research settings. 